\title{
Human Dental Pulp Stem Cells - A Prospective Regenerative Therapy
}

\author{
Namrata Tiwari \\ Research Scholar, School of Pharmacy, Babu Banarsi Das University, Lucknow, Uttar Pradesh, India
}

\begin{abstract}
Stem cells are the pluripotent cells that possess the potential to differentiate into a variety of cells of the human body. The knowledge of stem cell is enhancing not only in the medical field but equally in the dental field too. The stem cells of the dental origin provide remedies to many cell-based therapies in the regenerative medicine. Dental pulp stem cells (DPSCs) possess the mesenchymal stem cells phenotype and therefore get differentiated into neuron, chondrocytes, cardiomyocytes. The demand of cry preserving human exfoliated deciduous teeth (SHED) cells or the DPSCs are gaining popularity, in a similar fashion of the umbilical cord blood storage. The dental pulp stem cells are the adult multipotent cells that thrive in the cell abundant zone of the dental pulp. Accordingly, this multipotent characteristic makes the DPSCs an apt model to be incorporated in both dental and medical applications respectively. Therefore, this review which has been executed by both the electronic and hand-searching tools provides an outline of the applications of the dental pulp stem cells in the treatment of various medical disorders, nevertheless other aspects like the long-term clinical trials and studies needs more investigation to ascertain their efficacy.
\end{abstract}

Keyword: Dental Pulp Stem Cells, Tissue engineering of Dental Pulp Stem cells, Isolation of Dental Pulp Stem Cells, Cryopreservation of Dental Pulp stem cells, human exfoliated deciduous teeth (SHED)

\section{INTRODUCTION}

\section{PHYSIOLOGY OF THE TEETH}

The teeth are characterized by the cells of which it is formed, namely the buccal epithelial cells which form the enamel organ and subsequently the mesenchymal cells which take into form of the dental papilla. The enamel is made up by the enamel organ, and the dentin is made up by the dental papilla. In the tooth formation, the cells from the neural crest also take an active participation $(1,4)$. These cells are formed in the nervous system and then later on they migrate to the maxilla and the mandible, where they interact with mesenchymal cells in order to form the enamel organ and the dental papilla $(2,3)$.

The tooth comprises of two anatomical parts, namely the crown and the root. The crown is the part of the tooth which is covered with enamel and it is the part which is usually visible in the mouth. The root is embedded deep inside the jaw. It gives anchorage to the tooth in its bony socket and is usually not visible. The tissues of the tooth are enamel, dentin, cementum and pulp. The blood vessels and the nerves are present in the pulp which enters the tooth from a hole at the apex of the root and cementum (1). The periodontal ligament which is at the vicinity of the tooth attaches the cementum to the alveolar (4).

\section{Dental Pulp Tissue}

Dental pulp is a heterogeneous soft tissue located in the center of the teeth that comprises of a variety of cell types and extracellular matrix molecules (5). The major cell of this tissue is the odontoblast which is also referred as the dentinoblast. The dental pulp comprises of the fibroblasts, undifferentiated mesenchymal cell or stem cells, macrophages, and lymphocytes (6).

\section{Periodontal Ligament (PDL)}

The periodontal ligament (PDL) is a vascularised, cellular soft connective tissue that encapsulates the teeth and connects the root cementum with the hard sheet of the alveolar bone (7). The cells which are in the PDL are generally the fibroblasts that primarily have a function to synthesize and maintain the 
extracellular matrix. These fibroblasts contain a welldeveloped cytoskeleton of microtubules and actin microfilaments which might have been implicated in cellular motility activities. Moreover, apart from fibroblasts, the PDL consists osteoblasts, osteoclasts, cement oblasts, macrophages, and stem cells which are capable of generating fibroblasts, cement oblasts, and osteoblasts $(8$,$) .$

\section{STEM CELLS}

The term stem cell was coined for scientific purpose by Russian histologist Alexander Maksimov in 1909. Alexander Maksimov was the first to put forward the existence of hematopoietic stem cells (HSC) with the morphological appearance of a lymphocyte, capable of migrating throughout the blood to microecological niches that would allow them to proliferate and differentiate (3).

Stem cells are the biological cells that possess two properties: self-renewal, i.e. the ability to go through unlimited cycles of cell division for the cause of replenishing the cell pool, and the potency, i.e. the capacity to differentiate into other cell types. Depending on the cells' proliferative potential, the available pool of stem cells does not decrease when the cells are transformed into other tissues and instead they participate in the renewal and the replenishment of the vital organs such as the bone marrow $(1,5)$.

A zygote is the initial, ideal stem cell. A zygote is regarded as a tot potent cell that has the capacity to develop into mature cell. Many huge numbers of stem cells are observed during fetal development. When the embryo develops, stem cells are basically deprived of their capacity to transform into other cells and thus differentiation may take place only in a given germ layer. For unlimited division, the embryo's capacity is preserved until stem cells differentiate into three germ layers (2).

The cells that are transformed solely into endoderm, ectoderm and mesoderm layer cells are collectively known as multipotent stem cells. Unipotent stem cells have the potential to develop into only one type of cell (7). Pluripotent stem cells are those that can give rise to every cell of an organism except its extraembryonic tissues, the placenta. This drawback restricts the pluripotent stem cells from developing into a full organism. Embryonic stem (ES) cells and induced pluripotent stem (iPS) cells are pluripotent stem cells. Multipotent stem cells are adult stem cells which only generate specific lineages of cells $(2,6)$.

A recently occurred development, with a remarkable significance for clinical therapy has been the generation of induced pluripotent stem (iPS) cells from somatic cells. The method used for the iPS cell induction was termed as "ground breaking" because somatic cells were converted directly into pluripotent cells through introduction of four genes: Oct-4, Sox-2, c- Myc and Klf4 (7).

Today, stem cell biology is considered as one of the most fascinating areas of science which promises a ray of hope in the form of improved outcomes by replacing damaged or absent tissues with healthy regenerated tissue.

\section{Mesenchymal stem cells}

Alexander Friedentstein was the first to decipher the presence of a population of no hematopoietic cells that possessed the capability of auto renovation and bone differentiation in the bone marrow (9). Moreover, the research laid down by other investigators gave evidence that the bone marrow derived cells which were isolated as per the Friendenstein's techniques, also possessed high potency of proliferation and pluripotency of differentiation into mesenchymal tissues, and hence Caplan used the term "mesenchymal stem cell" (MSC) to describe them. The other studies put forward further investigations that the mesenchymal stem cells are characterized as a heterogeneous cell population wherein each individual possesses different characteristics and varies in its gene expression, differentiative capacity, expansion potential and phenotype. Since all of them do not follow the stem cell criteria. Hence, they are preferred to be called "multipotent stromal cell" with the same abbreviation "MSC" (42).

Various studies have produced information that MSCs can be isolated from various multiple tissues, such as bone marrow, peripheral blood, umbilical cord blood, adult connective tissue, dental tissues, placenta and amniotic membrane (9).

Today, any population of cell that meets the following attributes, irrespective of their source, is generally referred to as MSC: morphologically, they have the tendency to adhere to plastic and have a fibroblastlike appearance; functionally, they have the ability of 
self-renewal and could differentiate into cells of the mesenchymal lineage (osteocyte, chondrocyte and adipocyte), and into the cells of the endoderm (hepatocytes) and ectoderm (neurons) lineages under suitable cell culture conditions; phenotypically, they express more than $95 \%$ of the population express the CD105, CD73, CD90 surface antigens and that less than $2 \%$ of the population of the cells express the panleukocyte marker CD45, the hematopoietic progenitor and endothelial cell marker CD34 (44).

\begin{tabular}{|c|c|c|c|c|c|}
\hline \multicolumn{6}{|c|}{ ypes of Stem Cell in dental pulps } \\
\hline Characteristics & DPSC & SHED & SCAP & PDLSC & DFPC \\
\hline Site & Permanent tooth pulp & $\begin{array}{c}\text { Exfoliated deciduous } \\
\text { tooth pulp }\end{array}$ & $\begin{array}{l}\text { Apical papilla of } \\
\text { developing root }\end{array}$ & Periodontal ligament & $\begin{array}{l}\text { Dental follide of } \\
\text { developing tooth }\end{array}$ \\
\hline Proliferation Rate & Moderate & High & High & High & High \\
\hline Mutli-potentiality & $\begin{array}{c}\text { Odontoblast, } \\
\text { osteoblast, } \\
\text { chondrocyte, } \\
\text { neurocyte, adipocyte, } \\
\text { comeal epithelial cells, } \\
\text { induced pluripotent } \\
\text { stem cells }\end{array}$ & $\begin{array}{c}\text { Odontoblast, } \\
\text { osteoblast, } \\
\text { chondrocyte, myocyte, } \\
\text { neurocyte, adipocyte, } \\
\text { induced pluripotent } \\
\text { stem cells }\end{array}$ & $\begin{array}{c}\text { Odontoblast, } \\
\text { osteoblast, neurocyte, } \\
\text { adipocyte, induced } \\
\text { pluripotent stem cells }\end{array}$ & $\begin{array}{l}\text { Odontoblast, osteoblast, } \\
\text { chondrocyte, neurocyte }\end{array}$ & $\begin{array}{l}\text { Odontoblast, } \\
\text { osteoblast, } \\
\text { neurocyte }\end{array}$ \\
\hline Tissue repair potential & $\begin{array}{l}\text { Bone regeneration, } \\
\text { neuroregeneration, } \\
\text { dentin pulp } \\
\text { regeneration }\end{array}$ & $\begin{array}{l}\text { Bone regeneration, } \\
\text { neuroregeneration, }\end{array}$ & $\begin{array}{l}\text { Bone regeneration, } \\
\text { neuroregeneration, } \\
\text { dentin pulp } \\
\text { regeneration, root } \\
\text { formation }\end{array}$ & $\begin{array}{l}\text { Bone regeneration, root } \\
\text { formation }\end{array}$ & $\begin{array}{c}\text { Bone regeneration, } \\
\text { periodontal } \\
\text { regeneration }\end{array}$ \\
\hline
\end{tabular}

\section{SOURCE OF CELLS}

The most anticipated cell sources for tissue engineering are the stem cells. At present, umpteenth types of adult stem cells have been isolated from teeth, including dental pulp stem cells (DPSCs) $(3,8)$, stem cells from human exfoliated deciduous teeth (SHEDs), periodontal ligament stem cells (PDLSCs), dental follicle progenitor stem cells (DFPCs), and stem cells from apical papilla (SCAPs) $(5,46)$.

Mesenchymal Dental Stem Cells (MDSCs) are basically multipotent cells which has the capacity to proliferate extensively (maintained for at least 25 passages), can be safely cryopreserved, they even then possess immunosuppressive properties, and even exhibit mesenchymal markers. Moreover, the stem cells which are obtained from the teeth are morphologically large spindle-shaped cells with a large central nucleus abundant cytoplasm, and cytoplasmic extensions in the culture. Such adherent cells are morphologically identical to the mesenchymal stem cells which are obtained from bone marrow (BMMSCs). MDSCs are capable of differentiating in vitro into the cells of all of the germinal layers, namely ectoderm (neural cells), mesoderm (myocytes, osteoblasts, chondrocytes, adipocytes, and cardiomyocytes), and endoderm (hepatic cells) (41).

\section{ISOLATION OF THE DENTAL PULP STEM CELLS}

The extracted teeth by the routine dental practice and exfoliated deciduous teeth by physiological resorption are the two dental pulp sources. The isolation process of the DPSCs is quite easy and is fully non-invasive. The dental crown is disinfected prior before extraction with $0.3 \%$ chlorhexidine and after extraction with iodine and $70 \%$ ethanol. If the teeth are being transported to other lab then it must be kept in a transport medium. The transport medium should include at least $3 \%(\mathrm{w} / \mathrm{v})$ penicillin and streptomycin and $5 \mu \mathrm{g} / \mathrm{ml}$ amphotericin B (7). Permanent teeth should be crushed with a mini hammer in order to get a view of the pulp chamber and root canals. Once after breaking, the crowns of the resorbed deciduous teeth have been crushed, then a clear view is established, the pulp tissue can then be taken out with toothless surgical pliers (15) 


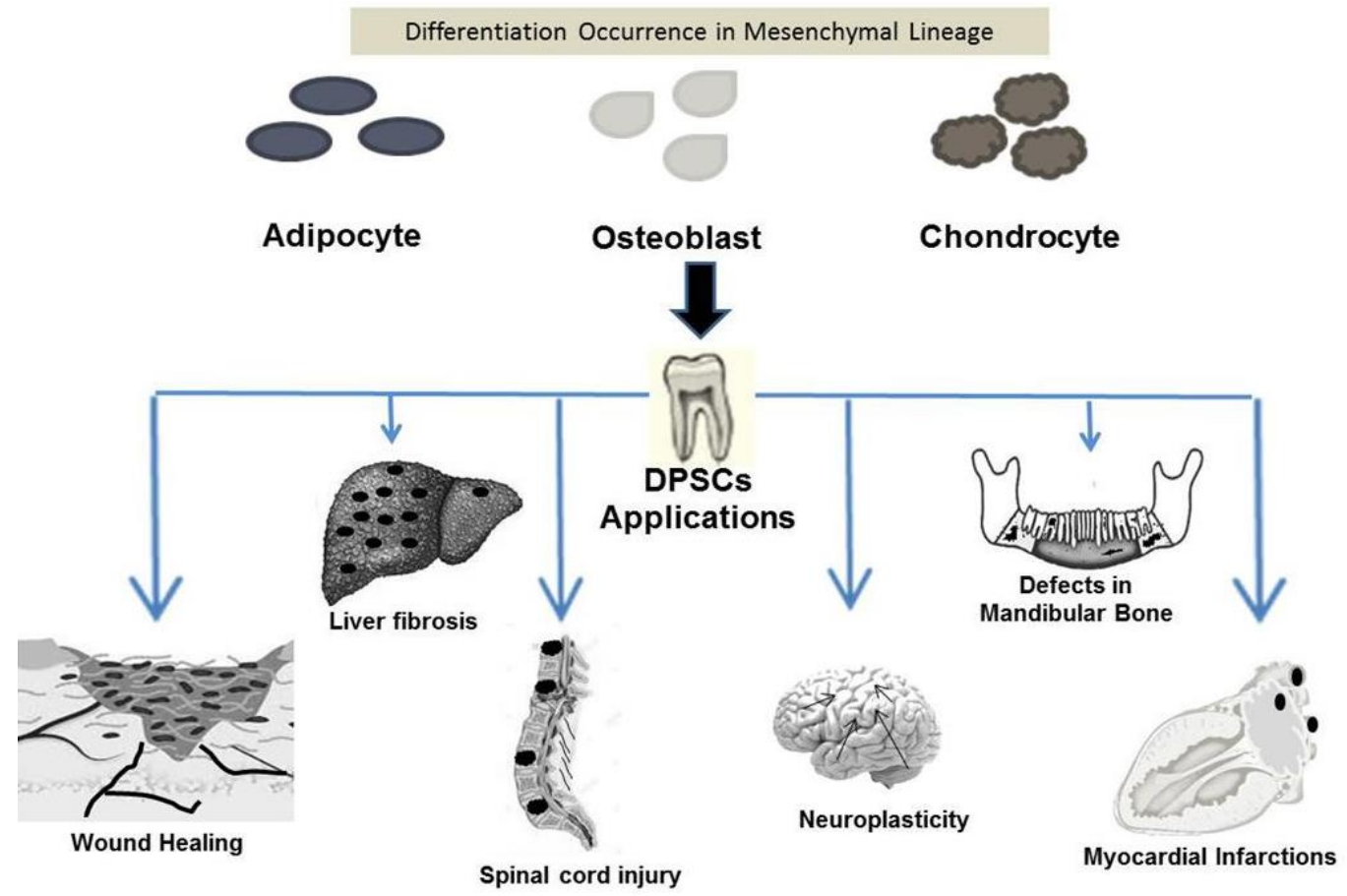

Figure 1: Multilineage Differentiation Potential and Clinical Applications of Dental Pulp Stem Cells

There are mainly two isolation methods-

1. Enzyme digestion

2. Outgrowth method

Enzyme Digestion- The pulp tissue is washed with sterile phosphate buffer (PBS) and it is then cut into small fragments $\left(\sim 1 \mathrm{~mm}^{\wedge} 3\right.$ pieces $)$. The tissue pieces obtained should be washed twice with sterile PBS and placed in a solution of $2 \mathrm{mg} / \mathrm{ml}$ collagenase type I and dispase for $30-45 \mathrm{~min}$ at room temperature, with gentle agitation on an orbital shaker. The cell suspensions can be obtained by passing the tissue through a strainer. The homogenate obtained is then centrifuged and the pellet is then resuspended in the growth medium and further it can then be cultured and expanded up to several passages.

Outgrowth method- In this method, the pulp tissue after shortening it into small fragments, it is then directly placed in a cell culture plate with a growth medium. The study produced by Nakashima illustrated that four different enzymatic separation (trypsin, collagenase with and without trypsin treatment, and a trypsin-collagenase mixture) did not result any effect on the morphological characteristics of the pulp cells which has been cultured, but the trypsin pretreatment/collagenase method produced the highest number of isolated cells. One report states that the enzymatic method results into different cell types to develop and the study also stated that the morphologies of the colonies obtained are spindle, stellate and epithelial like cells whereas the other group stated that compact and loose colony types were the end result of the morphological change, when the enzyme digestion method was exhibited $(19,21,22)$.

\section{STEM CELL BASED TISSUE ENGINEERING OF DENTAL PULP STEM CELLS}

The term "tissue engineering" was coined by Langer and Vacanti in the early 1990s for describing the technique for biological tissue regeneration (12). Tissue engineering approach requires three main key elements (triad): Stem cells, Scaffolds (matrix) and growth factors (morphogens) (11).

Tissue engineering is a potential resolution for tissue/organ replacements and has been feasible by the cooperation of biological and material sciences. The technique basically involves the use of ex vivo expanded cells grown on a support of biocompatible material under appropriate environmental conditions in order to create tissue replacement and living prostheses (10).

Tissue engineering is an interdisciplinary field of study that applies the principles of engineering to biology and medicine towards the development of biological substitutes that are responsible for restoration, maintenance, and overall improvement of the normal functions (12). Hence, the emerging discipline of the tissue engineering and regenerative 
medicine highlights the use of a rational approach which is based on morphogenetic signals for induction of tissue, responding stem/ progenitor cells and the scaffold to protect and save the natural flora of the microenvironment (12). At present, there are two basic branches of research in the area of the tooth tissue regeneration. The first branch deals with the restoration of the partial tooth damages. This stream of research is inclined towards using existing reparative capacities of the tooth and/or use of the tooth-related stem cells for repair of damaged tooth parts. Whereas, the second stream focuses on the whole regeneration by the incorporation of stem cells and conventional tissue engineering techniques (27). Such researches which highlights on the whole tooth regeneration bifurcated into two main directions: (1) in vitro cell culture and in vivo implantation (2) in vivo implantation of engineered cells (25).

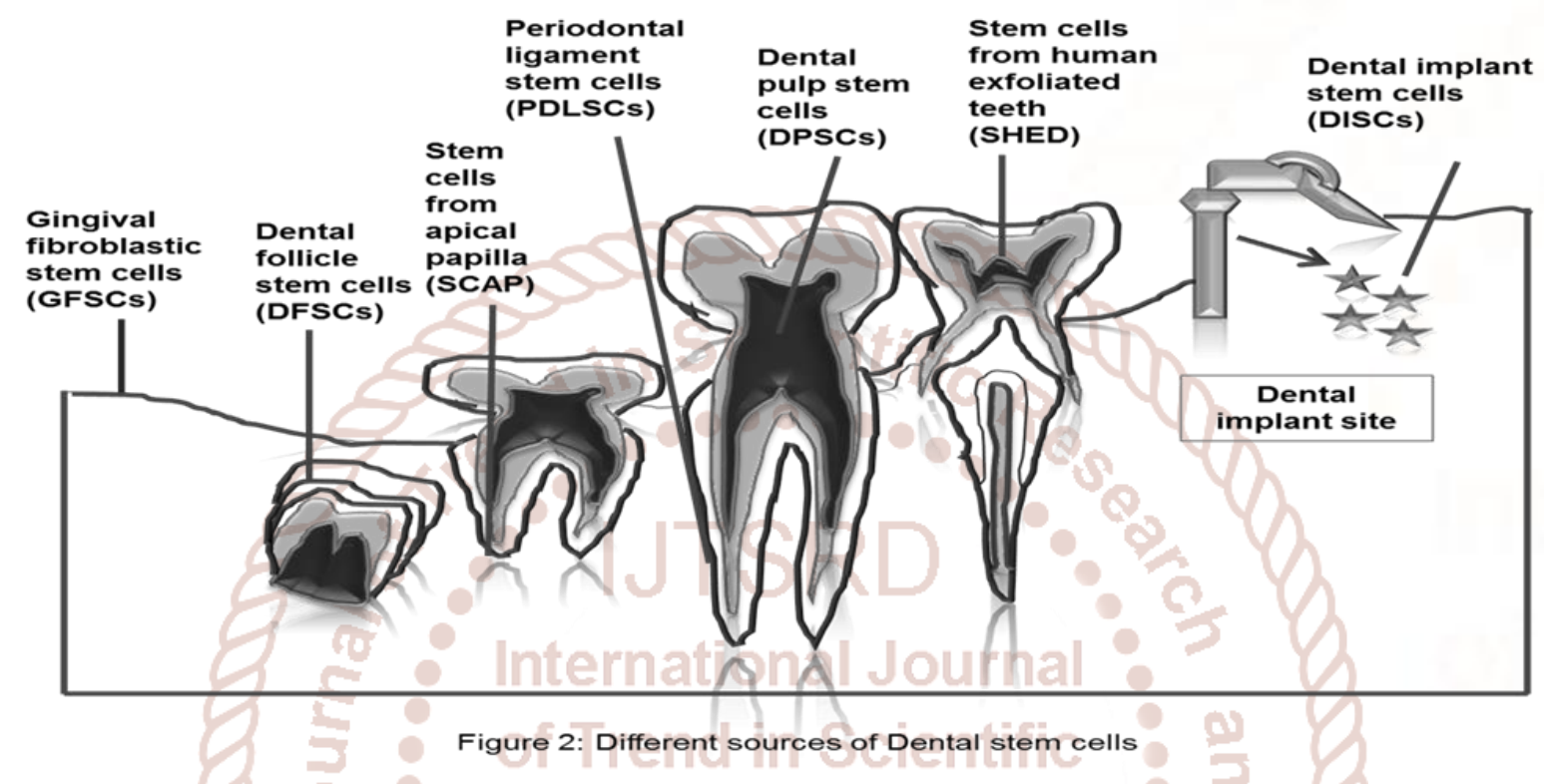

Due to the ongoing researches it is now evident that the tooth crowns can be regenerated provided there is good amount of suitable conditions, such as the in vitro organ culture, grafts on chick chorioallantoic membrane, ocular grafts, subcutaneous transplants, or kidney capsules. Apart from this, it is essential to create a three-dimensional (3D) organization of cells, to support the differentiation of these cells, and to avoid the xenograft rejection (12).

There were attempts to make a bioroot which showed some positive results. When the blocks of the hydroxyapatite/tricalcium phosphate (HA/TCP) containing SCAP and PDLSC are inserted into the socket of swine it led to the regeneration of the root/PDL complex, on top of which an artificial dental crown can be affixed. By this way, engineered bioroots showed lower compressive strength than that of the natural swine root dentin, but it is noteworthy that they resulted in normal functioning devoid of major hindrance and very well adjusted the porcelain crown (10).

The implantation of PDSLC seeded on HA/TCP is surgically created periodontal defects in miniature swine exhibited their excellent capacity to form bone, cementum, and PDL. These results hold utmost importance because of the high alarming statistics of periodontitis which is the leading cause of the tooth loss, especially in the elderly population (11). The tooth like structures can be created from biodegradable polymer scaffolds (collagen coated polyglycolic acid, calcium phosphate material, collagen sponges) seeded with dissociated single-cell suspension. These obtained structures comprises of enamel, dentin, and pulp, but some of the essential elements are not included, such as the complete root and periodontal tissues which allow correct anchoring into the alveolar bone, and which does not reach the expected size or shape of the scaffold $(12,13)$.

\section{SCAFFOLDS}

Another important component of tissue engineering is the scaffolds. The most suitable scaffolding material should support the attachment, proliferation, and differentiation of the seeded stem cells. Even if the strategy is used for dental pulp regeneration, an ideal scaffold must support vascularization and innervations of the pulp tissue. The investigators are in search for the "ideal scaffold" which possesses the attributes of facilitating the growth, integrating and differentiating the cells. Other essential prerequisites 
are that the scaffold should be biocompatible, nontoxic and should possess optimal physical features and mechanical properties. The best prognosis in the field of dental pulp regeneration, a regenerated highly vascularized soft tissue core with surrounding hard tissue should be incorporated. It has been noted that the experiments wherein the cell's free scaffolds are taken as a subject accounts for attractiveness because it provides an easier handling process that eliminates the risks which arises from issues that are associated with the use of the stem cells, with the storage and with its shelf-life, cost aspects, and immunoresponsiveness of the host as well as the transmission of the diseases (25). But there are few disadvantages too which are attached to this strategy which includes, the cells could have the possibility of possessing the low survival rates. Secondly, the cells can migrate to different locations within the body which could result in aberrant patterns to mineralization. A quick fix to this problem is to apply the cells together with a scaffold as then this would help in strengthening the position as well as in the maintenance of cell localization (26).

There are various materials which are designed and constructed for tissue engineering approaches, which are natural and synthetic polymers or inorganic materials and composites, which have been fabricated into porous scaffolds, nanofibrous materials, microparticles and hydrogels. The natural components used are collagen, elastin, fibrin, alginate, silk, glycosaminoglycans such as hyaluronan, and chitosan (27). The use of these materials provides a high degree of structural strength and they are basically compatible with the cells and the tissues and are biodegradable, but they do own the difficulty of processing and are afflicted with the risk of transmitting animal-associated pathogens or provoking an immunoresponse. The synthetic polymers such as the poly lactic acid (PLA), poly glycolic acid (PGA), and their copolymer, poly lacticco-glycolic acid (PLGA) they provide excellent chemical and mechanical properties which allow high control over the physicochemical characteristics, such as the molecular weight, configuration of polymer chains, or the presence of functional groups. Currently, the hydrogels have been explored for tissue engineering applications in great extent. The current use of hydrogels exhibits various exploring properties which includes high biocompatibility, tissue type water content and mechanical attributes which are similar to those of native tissue (28).

\section{GROWTH FACTORS}

Growth factors are considered as an extensive group of proteins that can induce cellular proliferation and differentiation by binding to receptors on the cell surface. A numerous variety of the growth factors have generously been used for dentin complex regeneration, including Transforming Growth Factors (TGFs), Bone morphogenetic proteins (BMPs), Platelet-derived growth factor (PGDF), Insulin like growth factor (IGF), and fibroblasts growth factors (FGFs). Out of these, BMP-2, BMP-4, BMP-7 have been shown to direct the pulp progenitor/stem cell differentiation into odontoblasts and result in dentin formation, and thus making the BMP family the most likely candidate for dental clinic applications (14). Bone Morphogenetic Proteins (BMPs) family members are used sequentially and repeatedly throughout embryonic tooth development, initiation, morphogenesis, cytodifferentiation and matrix secretion (29). Six different Bmps (Bmp2-Bmp7) are coexpressed temporally and spatially Bmp6 were identified in human primary culture of dental pulp cells. BMPs have been successfully applied for the regeneration of periodontal tissue and other factors, such as PGDF, IGF-1, FGF-2, TGF- $\beta$, and BMPs have utility in the tooth tissue engineering (30).

The third most essential factor for tissue engineering is to carefully select appropriate growth factors. As stated above, morphogens such as the BMPs can induce DPSCs to differentiate into osteoblast-like cells. Hence, the foremost challenge that should be taken into account is the facts that how can one deliver the growth factors effectively. Therefore, the direct application of growth factors has always resulted into temporary release. The limited half-life and unstable release of growth factors are unfavourable for the new tissue formation. When the studies have been compared to protein therapy, then a prudent decision came of using the gene therapy as a promising tool for eradicating out such difficulty. A study done on the mouse dental papilla cells, wherein they were transfected with growth/differentiation factor 11 (GDF 11) were demonstrated to express dentin sialoprotein (Dsp); osteo-dentin formation during pulpal wound healing was observed in dog teeth in vivo after Gdf11 electroporation. The same group was then treated up with GDF11 ultrasoundmediated gene delivery using microbubbles, demonstrating complete reparative dentin formation in animal model in vivo. The effectiveness and productivity of such kind of in vivo gene therapy 
highly depends on the vitality of the remaining dental pulp cells. Ex vivo gene therapy, involving the transfer of in vitro transfected cells back in vivo, may provide a better remedy $(14,29,31)$.

A covered experiment on a tooth slice model has been successfully used to analyze repair of the dentin-pulp complex. This model has been reanalyzed to study dental pulp regeneration. The utmost approach for this model is to fill the center void of the tooth slice with a biodegradable scaffold, followed by concurrent seeding with dental stem cells. When it is implanted in vivo, the seeded dental stem cells were able to differentiate into odontoblasts and endothelial-like cells. Even though, it is still hazy whether this thin tooth slice model can be a successful model to regenerate a full sized, vascularised dental pulp tissues for clinicalapplications. Restriction in the blood supply is a major concern for de novo pulp regeneration. In a recent study, a modified model was developed, which used a human tooth root fragment (6-7mm long), with an enlarged root canal (1.0-1.25mm wide), with one end sealed to mimic a natural tooth root. The dental stem cells were seeded onto a poly-D,L- lactide and glycolide (PLG) scaffold, which was then inserted into the fabricated tooth root. Then the cell-seeded tooth fragments were transplanted subcutaneously and harvested after three to four months. Analyses of the harvested implants revealed the formation of a well- vascularized soft tissue in the root canal space and a continuous layer of dentin like tissue which was lined with odontoblast type cells. Such results when obtained exhibited the potency of the root fragment model for the tooth regeneration (30). In spite of the subcutaneous surrounding is quite different from the alveolar bone, hence, the feasibility of incorporating such model for implanting into the jaw bone of the sheep and the minipig animal models requires further research and is still being counted as a base for further investigations.

\section{TOOTH CRYOPRESERVATION/BANKING}

The teeth which are extracted are generally thought to be medical waste. When intensive research was not carried out in this field then the therapeutic potential of such dental SCs was not well understood and there were no storage methods for potential donor teeth or SCs. Various types of dental SCs have now been identified from human teeth and surrounding tissues. When there came a protective means to preserve the dental SCs for medical applications, the term "tooth bank" was first raised 1966. And with the rapid development of advanced cryopreservation technology, the first commercial tooth bank was established as a venture company at National Hiroshima University in Japan in 2004. And as such, by various synchronised methods, an increasing number of teeth have been cryopreserved for future general medicine (10).

"Cryo" means cold in Greek, and cryopreservation is a process in which cells or whole tissues are preserved by cooling to subzero temperatures, typically $-196^{\circ} \mathrm{C}$. The formation of ice nucleation and the growth of a crystal structure are the main limitations of traditional cell/tissue preservation because these processes cause cell death. As such, vitrification, offers a relief and is considered as a better method of preserving tissues. Vitrification converts a material into a glass like amorphous solid without crystalline structure. Hence, for this reason, it can be used as a tool in protecting cells and tissues from dying during cryopreservation. There are basically three critical factors that affect vitrification: (1) Cooling and thawing rates: two cooling rates are reported when freezing. One is the conventional slow freezing method; the other is vitrification with an ultra high freezing rate. For the thawing rate, a rapid warming method is recommended. As heat is transferred from the external environment inwards when thawing, a slow warming rate causes the outside part to defrost first, but the central part is still cold enough to freeze the outside part again. This is likely to damage the cell. Therefore, rapid cooling and thawing rates are highly recommended. 2) Concentration of the cryoprotectant: the chemical toxicity and potential for osmotic injury by the cryoprotectant are very essential for cell viability. However, vitrification requires a high concentration of the cryoprotectant, which is a concern when preserving functional cells and tissues. (3) sample size and the carrier systems: reducing the sample and carrier sizes allow higher cooling and thawing rates $(11,13)$.

There is an abundant source of adult stem cells in the human exfoliated deciduous teeth (SHED). The recent studies have shown that SHED has the ability to develop into more types of body tissues than other types of stem cells.

Advantages of the SHED banking

1. it provides an autologous transport for life.

2. it renders simple and painless procedure. 
3. SHED cells are complementary to stem cells from the cord blood.

4. they are useful for close relatives of the donor.

5. they are not subjected to the same ethical concerns as embryonic stem cells.

A new way to cryopreserve DPSC inside a whole tooth was proposed by Silvia and associates. They exhibited some eye catching characteristics making them to supersede from any other method by the way that they showed a reduced price of the initial costs and workload of the tooth banking and even showed the avoidance of purification of the cells before cryopreservation (11).

There are various stem cell banks, storages that preserve and maintain ethically sourced stem cell obtained from different origins. The "International Society for Stem Cell Research and the International Stem Cell Banking Initiative" is one of the pioneers for this program (10). In India, "Life Cell International", was the first private sector stem cell banking services started in Bangalore (India) in the year 2009 .

Earlier, through individual and corporate approaches, charges for collecting and saving the stem cells were hardly within the reach (approximately 3000 USD); this has been the reason as to why it could not receive much attention and promotion in India (13).

Some of the licensed tooth stem cells banks, internationally and in India those of which uses cryopreservation and isolation are as follows $(12,13)$ :

1. In Japan, the first tooth bank was established in Hiroshima University and the company was named as "Three Brackets" (Suri Buraketto).

2. BioEden (Austin, Texas), StemSave, and a Storea-Tooth (USA)

3. The Norwegian tooth bank

4. In India, Stemade Biotech Pvt. Ltd (Delhi, Chennai, Chandigarh, Pune, and Hyderabad).

\section{WNT/B-CATENIN \\ SIGNALING IN DEVELOPMENT AND REGENERATION OF THE TOOTH}

Wnt proteins are evolutionary conserved secreted glycoproteins mediating short-range paracrine signaling. The name "Wnt" was coined post the discovery taht the Drosophila gene for wingless (wg) and the murine oncogene int-1 are conserved orthologues. Wnt proteins bind with serpentine receptors of the Frizzled (Fzd) family on cell membranes to trigger several distinct signaling cascades: the canonical or $\mathrm{Wnt} / \beta$ - catenin pathway; the Wnt/Ca+ pathway involving Protein Kinase A; the planar cell polarity pathway; and a pathway involving Protein Kinase $\mathrm{C}$ that functions in muscle myogenesis(32).Wnt $/ \beta$-Catenin pathway is active at multiple stages of tooth development as indicated by the expression of transgenic Wnt reporter genes (33). During the process of early tooth morphogenesis the Wnt / $\beta$-Catenin pathway is mainly active in dental epithelia, e.g dental lamina at initiation stage and enamel knots from cap to bell stage. For early tooth development epithelial Wnt $/ \beta$-Catenin signaling is essential (32). During later tooth development, Wnt / $\beta$-Catenin pathway is active in both differentiating dental epithelia and mesenchyme and concentrated around the developing roots in mice. In vitro, Wnt $/ \beta$-Catenin signaling pathway positively regulates the cement oblast/osteoblast differentiation of dental follicle cells. In mice, inhibition of mesenchyme Wnt / $\beta$-Catenin signaling pathway by Dkk1 overexpression impairs post-natal mandibular molar dentin formation, while the constitutive stabilization of $\beta$-Catenin in the dental mesenchyme leads to excessive dentin and cementum formation. Such findings suggested that mesenchyme Wnt $/ \beta$-Catenin signaling controls formation of dentin and local modulation of Wnt $/ \beta$-Catenin signaling has the therapeutic potential to improve the regeneration of these tissues (34).

\section{THERAPEUTIC APPLICATIONS}

Dental pulp tissue engineering is a promising field that can potentially have a major impact on the oral health (19). The dental stem cells can be isolated from different part of the teeth which are $(7,15)$ :

1. SHED

2. Adult dental pulp stem cells (DPSC)

3. Stem cells from the apical part of the papilla (SCAP)

4. Stem cells from the dental follicle (DFSC)

5. Periodontal ligament stem cells (PDLSC)

6. Bone marrow derived mesenchymal stem cells (BMSC)

7. Epithelial stem cells (EpSC)

8. Induced pluripotent stem cells (IPSC)

9. Immature dental pulp stem cells (IDPS)

10. Oral epithelial stem cells (OESCs)

11. Gingiva derived MSCs (GMSCs) 
12. Tooth germ progenitor cells (TGPCs)

13. Salivary gland stem cells (SGSCs)

14. Perisoteum derived stem cells (PSCs)

The dentistry has been devoted to the healing of defects with durable materials or the patient's own (autologous) tissue. The amalgam, composites and even titanium dental implants can fail, and all have limited service time. The stem cells from dental sources have found applications in treatment of various diseases and defects.

\section{Neural differentiation}

Various studies have shown that DPSCs have the capability of initiating ling term regeneration of nerves in damaged spinal cord. It was also reported that DPSCs expressed a neuronal phenotype and produced the neurotrophic factors like NGF (nerve growth factor), GDNF (Glial cell derived neurotrophic factor), BDNF (Brain derived neurotrophic factor) and BMP2. The DPSCs protected primary neurons and helped in the cell viability. The DPSCs promoted the regeneration of transacted axons by directly inhibiting multiple axon growth inhibitors and by prevention of apoptosis of neurons, astrocytes and oligodendrocytes. The DPSCs were then differentiated into mature oligodendrocytes to replace the cells that were lost $(16,37,38,39,40)$.

\section{Differentiation into bone cells}

The DPSCs are basically ectomesenchymal in origin and contain osteogenic markers which respond to the inductors of osteogenic and odontogenic differentiation. Such type of mesenchymal stem cells are extensively used in surgical repair/regeneration, as they initiate from neural crest and migrate, differentiate, participate in morphogenesis to give rise to structures of craniofacial region including muscle, ligament, cartilage, bone and teeth (47).

\section{Type 1 Diabetes}

Diabetes is one of the most common chronic degenerative endocrinal diseases which is related to the pancreatic islet cells that may possibly be successfully managed by transplantation of pancreatic islet cells. The embryonic and adult stem cells have been used for the production of insulin producing cells derived from amniotic fluid, bone marrow and adipose tissue. DPSCs have the capacity to differentiate into islet-like aggregates also shown by some groups $(43,44)$.

\section{Differentiation to hepatocyte}

The hepatocyte is a cells of the main tissue of the liver that makes up $70-80 \%$ of the liver's cytoplasmic mass. DPSCs has also shown to be differentiated into cells with morphological, phenotype and functional characteristics similar to hepatocytes. It has been further suggested that the stem cells derived have the capacity to differentiate into hepatic family $(37,38)$.

\section{In corneal production}

A study put forward its findings that a tissue engineered DPSC sheet when transplanted on the corneal bed and further on it was converted with the help of de-epithelialized human amniotic membrane, the histological analysis at three months postoperative phase confirmed that a healthy uniform corneal epithelium was formed. Thus, it was concluded that tissue engineered DPSC sheet was successful for the reconstruction of corneal epithelium $(18,45)$.

\section{In Ischemia}

The therapeutic application of DPSCs resulted in successful engraftment and an increase in the blood flow including high density of capillary formation in a rat model with hind limb ischemia (41).

\section{In myocardial infarction}

Studies have shown that DPSCs have been differentiated to cells with a cardiac phenotype. And the first person to detect and estimate it was Arninam and co-workers. They put forward that the tissue specific mesenchymal stem cells (MSCs) can change into cardiomyocytes $(35,45)$.

\section{Miscellaneous Work}

Other work done by other group stated that the DPSCs are useful in repair of myocardial infarction and that the human DPSCs secrete multiple proangiogenic apoptotic factors $(37,38)$.

\section{In muscular dystrophy}

When the immunochemistry (dystrophin markers) was carried out, it demonstrated through its findings that DPSCs had a significant engraftment in dog muscles. It was reported that the therapeutic potential of DPSC to differentiate into dystrophin producing multinucleated muscle cells can be successfully utilized in diseases like muscular dystrophy where body is incapable to produce dystrophin (47). 
International Journal of Trend in Scientific Research and Development (IJTSRD) ISSN: 2456-6470

\section{CONCLUSION}

Dental pulp is a remarkable site of stem cells and having been composed up of ectomesenchymal components containing neural crest-derived cells showcases its plasticity and multipotential capabilities. Their easy availability, preservation for longer period, lack of ethical/legal issues in its extraction, availability from the homogenous source, and multipotency are some of the advantages that make it a lucrative option over other types of stem cells (19).

Research stated that dental pulp is a remarkable site of stem cells; collecting stem cells from dental pulp is a non-invasive practice that can be performed in the adult during life and in the young after surgical extraction of wisdom teeth, a common surgical practice. Dental pulp is ideal for tissue engineering, for clinical use in several pathologies requiring bone tissue growth and repair. This group of researchers even stated that "if the bone marrow is considered as the site of first choice for hematopoietic stem cell collection, dental pulp must be considered as one of the major sites for mesenchymal cell collection" (44).

Hence, it can be rightfully said that the stem cells of the dental origin are easily procurable and have the capacity to differentiate into various lines, and not only regenerative endodontics but also in the treatment of many degenerative diseases that even highlights the true role of dental pulp stem cells.

\section{REFERENCES:}

1. Lee, S. M., Zhang, Q., \& Le, A. D. (2014). Dental Stem Cells: Sources and Potential Applications. Journal of Current Oral Health Reports, 1(1), 3442.

2. Ranganathan, K., \& Lakhsminarayan, V. (2012). Stem cells of the dental pulp. Indian Journal of Dental Research, 23(4), 558.

3. Casagrande, L., Cordeiro, M., Nor, S., \& Nor, J. (2011). Dental pulp stem cells in regenerative dentistry. Journal of Odontology, 99(1), 1-7.

4. Kostlan, J. (1965). Physiology of teeth and of the oral cavity in relation to dental caries. Stomatologiia, 44(5), 3-8.

5. Kabir, R., Gupta, M., \& Kola, M. (2014). Imperative Role of Dental Pulp Stem Cells in Regenerative Therapies: A Systematic Review. Nigerian Journal of Surgery, 20(1), 1-8.
6. Tayal, E. (2016). Dental Stem Cells: Part of Regenerative Medicine. International Journal of Preventive and Clinical Dental Research, 3(4), 277-281.

7. Tatullo, M., Marrelli, M., Shakesheff, K., \& White, L. (2014). Dental pulp stem cells: function, isolation and applications in regenerative medicine. Journal of Tissue Engineering and Regenerative Medicine, 11(9), 1205-1216.

8. Masthan, K. M. K., Sankari, S. L., \& Gopalakrishnan, T. (2013). Mystery inside the Tooth: The dental pulp stem cells. Journal of Clinical and Diagnostic Research. 7(5), 945-947.

9. Perry, B. C., \& Zhou, D. (2008) Collection, Cryopreservation, and Characterization of Human Dental Pulp-Derived Mesenchymal Stem Cells for Banking and Clinical Use. Journal of Tissue Engineering. 14(2), 149-156.

10. Huynh, N, C., Le, S. H., Doan, V. N., Ngo, L. T. Q., \& Tran, H. L. B. (2017). Simplified conditions for storing and cryopreservation of dental pulp stem cells. Archives of Oral Biology. 84, 74-81.

11. Han, Y., Kang, Y., Shivakumar, S., Bharti, D., Son, Y., Choi, Ho, et al (2017). Stem Cells from Cryopreserved Human Dental Pulp Tissues Sequentially Differentiate into Definitive Endoderm and Hepatocyte-Like Cells in vitro. International Journal of Medical Sciences. 14(13), 1418-1429.

12. Demarco, F. F., Conde, M. C. M., et al., (2011). Dental Pulp Tissue Engineering. Brazilian Dental Journal, 22(1), 3-13.

13. Eubanks, E. J., Tarle, S. A., \& Kaigler, D. (2014). Tooth storage, dental pulp stem cell isolation, and clinical scale expansion without animal serum. Journal of Endodontics, 40(5), 652-657.

14. Bonnamain, V., Thinard, R., Sergent-Tanguy, S., Huet, P., Bienvenu, G., et al (2013). Human dental pulp stem cells cultured in serum-free supplemented medium. Frontiers in Physiology.

15. Atari, M., Barajas, M., Hernandez, F., Gil, C., Fabregat, M, et al., (2011). Isolation of pluripotent stem cells from human third molar dental pulp. Histology and Histopathology. 26(8), 1057-70.

16. Lihua, L., Yan, H., Xiaoyan, W., Brian, K., Bae, H., et al., (2018). Potential Roles of Dental Pulp Stem Cells in Neural Regeneration and Repair. Stem Cells in International, 10(1155). 
17. Francesa, N., Anna, P., Pasqualina, S., Roberta, N., Montellaa, R., et al., (2014). Dental pulp stem cells: State of the art and suggestions for a true translation of research into therapy. Journal of Dentistry, 42(7), 761-768.

18. Fatima, N. S., Yipin, D., \& James, L, F. (2015). Dental Pulp Stem Cells: A New Cellular Resource for Corneal Stromal Regeneration. Stem Cells Translational Medicine, 4(3), 276-285.

19. Alessandra, P., Gianluca, C., \& Anto, D, P. (2015). Human Dental pulp stem cells (hDPSCs): isolation, enrichment and comparative differentiation of two sub-populations. Journal of Developmental Biology, 15 (14).

20. Suchanek, J., Soukup, T., Ivancakova, R., Karbanova, J., Hubkova, V et al., (2007). Human dental pulp stem cells-isolation and long term cultivation. Acta Medica, 50(3), 195-201.

21. Karamzadeh, R., Mohamadreza, B. E., \& Reza, A. (2012). Isolation, Characterization and Comparative Differentiation of Human Dental Pulp Stem Cells Derived from Permanent Teeth by Using Two Different Methods. Journal of Visualized Experiments, 69 (4372).

22. Federico, F., Renza, S., Antonio, P., Daniela, C., \& Francesco, C. (2012). Isolation and Characterization of Human Dental Pulp Derived Stem Cells by Using Media Containing Low Human Serum Percentage as Clinical Grade Substitutes for Bovine Serum. PlosOne, 10(1371).

23. Ana, A., Yvonne, P., \& Paul, T. S. (2010). Stem cell-based biological tooth repair and regeneration. Trends in Cell Biology, 20-206(126), 715-722.

24. Luciano, C., Mabel. C., Silvia, A., Jacques, E. (2011). Dental pulp stem cells in regenerative dentistry. Journal of Odontology, 99, 1-7.

25. Li, Z., Zhanga, Y., Morsib, Y., Wanga, Y et al., (2013). Review scaffold design and stem cells for tooth regeneration. Japanese Dental Science Review, 49(1), 14-26.

26. Evandro, P., Adriana, F., Jacques, N. (2014). Functionalized scaffolds to control dental pulp stem cell fate. Journal of Endodontics, 40(4), 3340.

27. Tran, H. L. B., Nguyen, M. T., Doan, V. N. (2015). Fabrication and evaluation of human dentin as scaffold for dental pulp stem cells.
Tissue Engineering and Regenerative Medicine, 12(4), 222-230.

28. Asghari, F., Salehi, R., Agazadeh, M., Alizadeh, E., Adibkia, K., et al., (2016). The odontogenic differentiation of human dental pulp stem cells on hydroxyapatite-coated biodegradable nanofibrous scaffolds. Journal International Journal of Polymeric Materials and Polymeric Biomaterials, 14(65).

29. Gorin, C., Rochefort, G., Bascetin, R., Ying, H., Lesieur, J. et al., (2016). Priming Dental Pulp Stem Cells With Fibroblast Growth Factor-2 Increases Angiogenesis of Implanted TissueEngineered Constructs Through Hepatocyte Growth Factor and Vascular Endothelial Growth Factor Secretion. Stem Cells Translational Medicine, 5 (3), 392-404.

30. Sowa. K., Nito, C., Nakajima, M., Ueda, M., Kimura, K., et al., (2018). Impact of Dental Pulp Stem Cells Overexpressing Hepatocyte Growth Factor after Cerebral Ischemia/Reperfusion in Rats. Methods and Clinical Development, 10, 281290.

31. Cào, X., Jin, S., Sun, L., Zhan, Y., Lin, F., et al., (2017). Therapeutic effects of hepatocyte growth factor-overexpressing dental pulp stem cells on liver cirrhosis in a rat model. Scientific Reports, 7.

32. Liu, F., and Millar, S. (2010). Wnt/ $\beta$-catenin Signaling in Oral Tissue Development and Disease. Journal of Dental Research, 89(4), 318330.

33. Tamura, M., and Nemoto, E. (2016). Role of the Wnt signaling molecules in the tooth. The Japanese Dental Science Review, 52 (4), 75-83.

34. Liu,F., Chu, E., Watt, B., Zhang, Y., Gallant, N., et al. (2008). Wnt $/ \beta$-catenin signaling directs multiple stages of tooth morphogenesis. Developmental Biology, 313(1), 210-224.

35. Mahla, R. (2016). Stem Cells Applications in Regenerative Medicine and Disease Therapeutics. International Journal of Cell Biology, 1155(10).

36. Bansal, R., and Jain, A. (2015). Current overview on dental stem cells applications in regenerative dentistry. Journal of Natural Science, Biology and Medicine, 6(1), 29-34.

37. Feng, R., and Lengner, C. (2013). Application of Stem Cell Technology in Dental Regenerative Medicine. Advances in wound care, 2(6), 296-305. 
38. Yap, M., Nathan, K., Heng, B. (2015). Neural Differentiation of Human Pluripotent Stem Cells for Nontherapeutic Applications: Toxicology, Pharmacology, and In Vitro Disease Modeling. Stem Cells International, 1155.

39. Goorha, S., and Reiter, L. (2017). Culturing and Neuronal Differentiation of Human Dental Pulp Stem Cells: Neuronal Differentiation of Human Dental Pulp Stem Cells. Current protocols in Human Genetics, 21(6).

40. Ariffin, S. H. Z., Kermani, S., Abidin, I., Wahab, R., Yamamoto, Z., et al. (2013). Differentiation of Dental Pulp Stem Cells into Neuron-Like Cells in Serum-Free Medium. Stem Cells International, 250740, 10.

41. Park, Y., Cha, S., Park, Y. (2016). Regenerative Applications Using Tooth Derived Stem Cells in Other Than Tooth Regeneration: A Literature Review. Stem Cells International, 9305986, 12.

42. Otsu, K., Kishigami, R., Sasaki, A., Harada, H. (2011). Differentiation of Induced Pluripotent Stem Cells into Dental Mesenchymal Cells. Stem Cells and Development, 21(7), 1156-64.
43. Abdulazeez, S. (2015). Diabetes treatment: A rapid review of the current and future scope of stem cell research. Saudi Pharmaceutical Journal, 23(4), 333-340.

44. Ezquer, M., Rodriguez, M., Billoud, M., Ezquer, F. (2014). Mesenchymal Stem Cell Therapy in Type 1 Diabetes Mellitus and Its Main Complications: From Experimental Findings to Clinical Practice. Journal of Stem Cell Research \& Therapy, 4(227).

45. Chalisserry, E., Nam, S., Anil, S. (2017). Therapeutic potential of dental stem cells. Journal of Tissue Engineering, 8(2041731417702531).

46. Krasner, A., Verlander, P. (2012). Stem Cells in Dentistry and Medicine: The Dentist's Role. Dental CE Today.com

47. Pisciotta, A., Riccio, M., Carnevale, G., Lu, A. (2015). Stem cells isolated from human dental pulp and amniotic fluid improve skeletal muscle histopathology in mdx/SCID mice. Stem Cell Research \& Therapy, 6, 156. 